\section{Tossing the baby out with the bathwater after a brief rinse? The potential downside of dismissing food addiction based on limited data}

\author{
Nicole M. Avena, Ashley N. Gearhardt, Mark S. Gold, Gene-Jack Wang \\ and Marc N. Potenza
}

A recent Perspective article that provided a critical analysis of the food addiction model as it relates to obesity stated that the model is "misleading", with supporting evidence described as "inconsistent and weak" (Obesity and the brain: how convincing is the addiction model? Nature Rev. Neurosci. 13, 279-286 (2012)) $)^{1}$. Whether non-drugrelated addictions exist has been debated, with recent proposals for the fifth edition of the Diagnostic and Statistical Manual of Mental Disorders (DSM-5) giving the concept greater credence ${ }^{2-4}$. The timely article by Ziauddeen et al. ${ }^{1}$ raised important points, including differences in findings across studies of obesity. Although we agree that not all obese individuals exhibit food addiction, we believe it is premature to reject the concept of food addiction. The extent to which food addiction applies to individuals with obesity, or subgroups thereof, deserves further research, particularly as such studies may help to better define clinically relevant subgroups who respond differently to specific prevention and treatment strategies ${ }^{5}$. Rejection of a model on the basis of limited data could be deleterious.

The investigation of clinically relevant eating-related behavioural phenotypes, including food addiction, has been cited as important ${ }^{6}$. As noted by Ziauddeen et al. ${ }^{1}$, preclinical models provide support for the construct of food addiction ${ }^{7,8}$. As in preclinical models, addictive eating patterns do not always equate to obesity in humans. When using the psychometrically validated Yale Food Addiction Scale that adapts DSM, fourth edition, text revision (DSM-IV-TR) criteria for substance dependence to food consumption, $25 \%$ to $37.5 \%$ of obese individuals meet criteria for food addiction, with rates for meeting the criteria being twofold to threefold times greater in obese versus lean groups ${ }^{9,10}$. Approximately fivefold greater rates of food addiction are observed in obese people with binge-eating disorder ${ }^{11}$, a condition that is responsive to different clinical interventions from those that are effective for non-binge-related obesity ${ }^{12}$.

Obesity is a heterogeneous entity. A failure to recognize and investigate individual differences could hamper the development of improved treatments and policies. Although Ziauddeen et al. ${ }^{1}$ note inconsistencies in addiction-related neurobiological findings in obesity, particularly those related to functional MRI, such findings may reflect the heterogeneity of obesity, as the authors indicate. Although the cognitive neuroscience approaches cited by the authors will be very important in the process of understanding this heterogeneity, they may be less transportable and adaptable to treatment settings than are clinical assessments, such as those measuring food addiction.

To determine the validity and clinical relevance of food addiction to obesity, or subgroups thereof, more research is needed, with the resulting findings being potentially relevant to efforts to match specific interventions with specific groups of people. Just as it might be premature to accept food addiction as a model for obesity, it may also be premature to discard such a model, particularly when so little research has been done (see TABLE 2 in Ziauddeen et al. ${ }^{1}$, citing only one functional MRI study that directly assesses food addiction). If found to be applicable to a considerable population with obesity, a food-addiction model may not only help clarify findings in obesity but, importantly, could also lead to new interventions for preventing and treating obesity, facilitated by decades of research in substance addiction $^{13}$. If we are to prematurely dismiss such a potentially important model on the basis of limited studies, we could miss important opportunities to improve health worldwide.

Nicole M. Avena is at the Department of Psychiatry,
McKnight Brain Institute, University of Florida,
Gainesville, Florida 32611 , USA, and the Department
of Psychology, Princeton University, Princeton, New Jersey 08544, USA.

Ashley N. Gearhardt is at the Department of Psychology, Yale University, New Haven, Connecticut 06520, USA.

Mark S. Gold is at the Department of Psychiatry, McKnight Brain Institute, University of Florida, Gainesville, Florida 32611, USA

Gene-Jack Wang is at the Medical Department, Brookhaven National Laboratory, Upton, New York 11973, USA.

Marc N. Potenza is at the Departments of Psychiatry and Neurobiology and Child Study Center, Yale University School of Medicine, New Haven, Connecticut 06519, USA.

N.M.A. and A.N.G. contributed equally to this work. Correspondence to M.N.P. e-mail: marc.potenza@yale.edu

doi: 10.1038/nrn3212-c1

1. Ziauddeen, H., Faroogi, I. S. \& Fletcher, P. C. Obesity and the brain: how convincing is the addiction model? Nature Rev. Neurosci. 13, 279-286 (2012).

2. Holden, C. 'Behavioral' addictions: do they exist? Science 294, 980-982 (2001).

3. Frascella, J., Potenza, M. N., Brown, L. L. \& Childress, A. R. Shared brain vulnerabilities open the way for nonsubstance addictions: carving addiction at a new joint? Ann. NY Acad. Sci. 1187, 294-315 (2010).

4. Holden, C. Behavioral addictions debut in proposed DSM-V. Science 327, 935 (2010).

5. Avena, N. M., Bocarsly, M. E., Hoebel, B. G. \& Gold, M. S. Overlaps in the nosology of substance abuse and overeating: the translational implications of "food addiction." Curr. Drug Abuse Rev. 4, 133-139 (2011).

6. Marcus, M. D. \& Wildes, J. E. Obesity: is it a mental disorder? Int. J. Eat Disord. 42, 739-753 (2009).

Avena, N. M., Rada, P. \& Hoebel, B. G. Evidence for sugar addiction: behavioral and neurochemical effects of intermittent, excessive sugar intake. Neurosci. Biobehav. Rev. 32, 20-39 (2008).

8. Kenny, P. J. Common cellular and molecular mechanisms in obesity and drug addiction. Nature Rev. Neurosci. 12, 638-651 (2011).

9. Davis, C. et al. Evidence that 'food addiction' is a valid phenotype of obesity. Appetite 57, 711-717 (2011).

10. Meule, A. How prevalent is "food addiction"? Front. Psychiatry 2, 61 (2011).

11. Gearhardt, A. N. et al. An examination of the food addiction construct in obese patients with binge eating disorder. Int. J. Eat. Disord. 30 Aug 2011 (doi: 10.1002/eat.20957).

12. Grilo, C. M., \& Mitchell, J. E. (eds.) The Treatment of Eating Disorders: A Clinical Handbook (Guilford Press, 2010)

13. Gearhardt, A. N., Grilo, C. M., DiLeone, J., Brownell, K. D. ¿ ¿ Potenza, M. N. Can food be addictive? Public health and policy implications. Addict. 106, 1208-1212 (2011).

Competing interests statement

The authors declare no competing financial interests. 